\title{
IMPACTO DE MÉTODOS DE SINCRONISMO NO DESEMPENHO DE GERADORES DE INDUÇÃO AUTO-EXCITADOS
}

\author{
Cassiano Rech* \\ cassiano@ieee.org \\ Maurício de Campos ${ }^{\dagger}$ \\ camposeunijui.edu.br \\ Gideon V. Leandro ${ }^{\dagger}$ \\ gede@unijui.edu.br
}

\author{
Robinson F. de Camargo ${ }^{\dagger}$ \\ robinson. camargo@unijui. edu.br \\ Fabiano Salvadori $^{\dagger}$ \\ f.salvadori@unijui.edu.br \\ Julio C. O. Bolacell ${ }^{\dagger}$ \\ bolacelleunijui.edu.br
}

*Núcleo de Processamento de Energia Elétrica - Universidade do Estado de Santa Catarina CEP 89223-100, Joinville, SC, BRASIL, +55-47-4009-7845

${ }^{\dagger}$ Grupo de Automação Industrial e Controle - Universidade Regional do Noroeste do Estado do Rio Grande do Sul CEP 98700-000, Ijuí, RS, BRASIL, +55-55-3332-0250

\section{RESUMO}

Este artigo analisa sistemas de controle digital, sem sensores mecânicos, usados para regular as tensões de saída de um gerador de indução com rotor gaiola de esquilo. Diferentes métodos de sincronismo são avaliados e seus desempenhos são comparados sob condições de carga distintas, incluindo cargas desequilibradas e não lineares. Resultados de simulação e experimentais são obtidos para demonstrar o impacto de sistemas de controle digitais distintos na distorção e no fator de desbalanço das tensões de saída.

PALAVRAS-CHAVE: Gerador de indução, método de sincronismo, regulação de tensão.

\section{ABSTRACT}

This paper analyzes digital sensorless control systems used to regulate the output voltages of a self-excited squirrel-cage induction generator. Different mechanical sensorless syn-

\footnotetext{
Artigo submetido em 05/12/2007

1a. Revisão em 29/07/2008

2a. Revisão em 10/10/2008

Aceito sob recomendação do Editor Associado

Prof. Enes Gonçalves Marra
}

chronization methods are evaluated and their performances are compared under several load conditions, including unbalanced and nonlinear loads. Simulation and experimental results are included to demonstrate the impact of distinct digital control systems on the output voltages distortion and the unbalance factor.

KEYWORDS: Induction generator, synchronization method, voltage regulation.

\section{INTRODUÇÃO}

Máquinas de indução podem gerar energia quando a velocidade do rotor é maior que a velocidade do campo magnético girante produzido pelo estator, e quando são conectadas a uma fonte externa de energia reativa. Em sistemas isolados, a energia reativa necessária para criar o campo magnético do estator pode ser fornecida por um banco de capacitores (Basset e Potter, 1935). Uma máquina de indução com rotor gaiola de esquilo é atrativa para sistemas de geração de baixa e média potência devido ao seu baixo custo, robustez, autoproteção contra sobrecarga e pela sua elevada densidade de potência $(\mathrm{W} / \mathrm{kg})$. Por outro lado, a amplitude e a frequiência das tensões de saída de um gerador de indução auto-excitado 


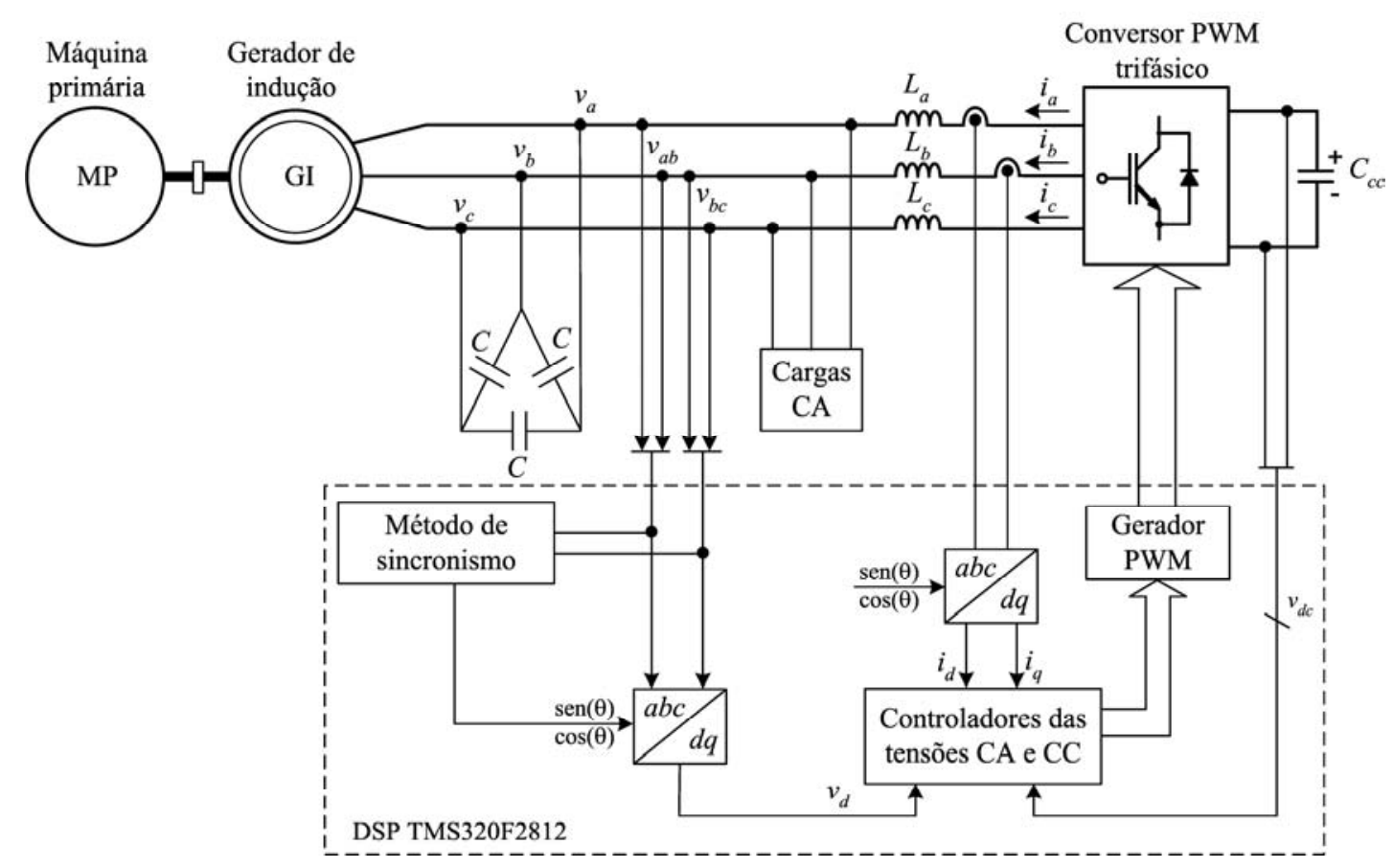

Figura 1: Diagrama simplificado do sistema de geração.

dependem da carga (Simões e Farret, 2008).

Várias soluções têm sido apresentadas para regular as tensões de saída de geradores de indução em sistemas isolados. Entre estas alternativas, é usual empregar um inversor alimentado em tensão com modulação por largura de pulso (PWM - Pulse Width Modulation), conectado em paralelo com o gerador de indução e as cargas CA (Ahmed et alli, 2006; Kuo e Wang, 2001; Leidhold et alli, 2002; Lopes e Almeida, 2006; Marra e Pomilio, 1999; Singh et alli, 2004). O inversor PWM opera como um compensador estático de reativos, que absorve ou injeta energia reativa no sistema de acordo com a carga para regular as tensões nos terminais do gerador de indução.

Diversas técnicas de controle têm sido desenvolvidas para controlar o inversor PWM conectado em paralelo e, conseqüentemente, para regular as tensões de saída sob diferentes condições de carga. Sistemas de controle digital sem qualquer sensor mecânico são particularmente interessantes, pois o custo de um sensor mecânico é significativo quando comparado ao custo de uma máquina de indução com rotor gaiola de esquilo para sistemas de geração de baixa e média potência (Ahmed et alli, 2006). Tais sistemas de controle são usualmente implementados nos eixos $d q$ para aplicações com máquinas elétricas trifásicas, uma vez que o problema de rastreamento é modificado para um problema de regulação, o que torna possível o uso de controladores do tipo proporcional-integral (PI) para regular as variáveis de inte- resse. Para converter as variáveis do sistema de coordenadas estacionário $a b c$ para o sistema de coordenadas síncrono $d q$ é necessário usar um método de sincronismo, que calcula os sinais de sincronismo empregados na matriz de transformação. Contudo, o impacto de diferentes métodos de sincronismo nas tensões produzidas por um sistema de geração isolado baseado em um gerador de indução auto-excitado ainda não foi apresentado.

Para preencher esta lacuna, este artigo analisa sistemas de controle digital, sem sensores mecânicos, usados para regular as tensões de saída de um gerador de indução auto-excitado. Diferentes métodos de sincronismo em malha aberta são avaliados e seus desempenhos são comparados sob diversas condições de carga, incluindo cargas desequilibradas e não lineares. Resultados de simulação e experimentais são incluídos para demonstrar o impacto de métodos de sincronismo distintos no fator de desbalanço e no conteúdo harmônico das tensões geradas.

Este artigo está organizado da seguinte forma: a Seção 2 mostra uma descrição básica do sistema sob estudo. A Seção 3 descreve os métodos de sincronismo investigados neste trabalho. A Seção 4 apresenta vários resultados para diferentes condições de carga e a Seção 5 inclui diversos resultados experimentais. 


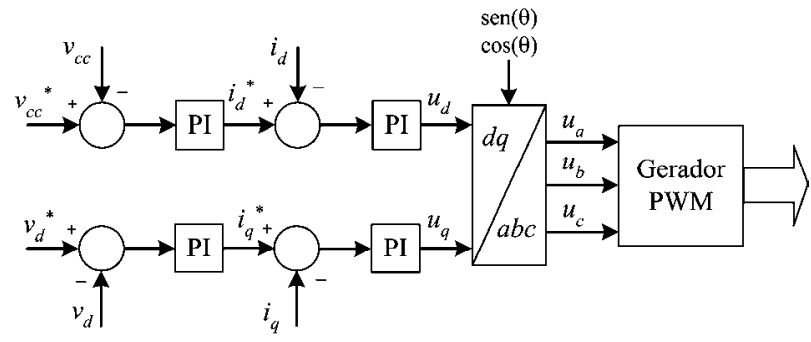

Figura 2: Diagrama de blocos dos controladores.

\section{DESCRIÇÃO DO SISTEMA DE GERA- ÇÃO}

Um diagrama simplificado do sistema sob estudo é mostrado na Figura 1. O sistema é composto por um gerador de indução com rotor gaiola de esquilo, excitado por um banco de capacitores trifásico, e por um inversor PWM alimentado em tensão. O barramento CC do inversor é composto por capacitores eletrolíticos, que atuam como fonte de tensão contínua. O gerador de indução é conectado ao lado CA do inversor através de indutores, que compõem um filtro de segunda-ordem trifásico com o mesmo banco de capacitores para reduzir harmônicos de alta freqüência produzidos pelo inversor.

O inversor PWM deve injetar ou absorver energia reativa para regular as tensões de saída do gerador de indução, independente das cargas conectadas no sistema. O inversor comporta-se como um banco de capacitores e injeta energia reativa no sistema quando as tensões de saída do gerador de indução são menores que a tensão de referência. Por outro lado, o inversor PWM absorve energia reativa do sistema de geração quando as tensões nos terminais do gerador são maiores que o valor desejado.

O sistema de controle digital sem sensores mecânicos empregado neste artigo usa o sistema de coordenadas síncrono $d q$. Portanto, as variáveis de realimentação no sistema $a b c$ devem ser transformadas para os eixos $d q$, usando a seguinte transformação:

$$
\mathbf{T}_{d q}=\left[\begin{array}{lll}
\frac{\sqrt{6}}{3} \cos (\theta) & -\frac{\sqrt{6}}{6} \cos (\theta)+\frac{\sqrt{2}}{2} \operatorname{sen}(\theta) & -\frac{\sqrt{6}}{6} \cos (\theta)-\frac{\sqrt{2}}{2} \operatorname{sen}(\theta) \\
-\frac{\sqrt{6}}{3} \operatorname{sen}(\theta) & \frac{\sqrt{6}}{6} \operatorname{sen}(\theta)+\frac{\sqrt{2}}{2} \cos (\theta) & \frac{\sqrt{6}}{6} \operatorname{sen}(\theta)-\frac{\sqrt{2}}{2} \cos (\theta)
\end{array}\right]
$$

De acordo com (1), um método de sincronismo deve ser utilizado para obter o ângulo de sincronismo $\theta$ ou os sinais seno e cosseno empregados na transformação $a b c$ para $d q$.
A Figura 2 apresenta um diagrama de blocos simplificado do sistema de controle do inversor PWM. O erro entre a tensão $\mathrm{CC}$ do barramento e seu valor de referência é a entrada de um controlador PI, que gera a corrente de referência no eixo $d\left(i_{d}^{*}\right)$. A corrente do inversor no eixo $d\left(i_{d}\right)$ controla o fluxo de energia ativa através do inversor PWM e, portanto, esta malha de controle afeta diretamente o nível de tensão do barramento CC. De forma similar, o erro entre a tensão de saída do gerador no eixo $d$ e seu valor de referência é o sinal de entrada de outro controlador PI, gerando a corrente de referência no eixo $q\left(i_{q}^{*}\right)$. A corrente do inversor no eixo $q\left(i_{q}\right)$ controla o fluxo de energia reativa através do inversor PWM, afetando a amplitude das tensões produzidas pelo sistema de geração. Os sinais de erro entre as referências de corrente obtidas das malhas externas de tensão e as correntes medidas são os sinais de entrada de controladores PI de corrente, que geram os sinais de controle no sistema de coordenadas $d q$.

Este artigo contribui com a análise de métodos de sincronismo usados na transformação $a b c$ para $d q$ e vice-versa. Portanto, como os mesmos controladores são empregados para todos os métodos de sincronismo apresentados a seguir, a análise e o projeto dos controladores não são apresentados neste artigo (Camargo e Pinheiro, 2005; Cichowlas e Kazmierkowski, 2002; Dai et alli, 2003; Erickson e Maksimovic, 2001).

\section{MÉTODOS DE SINCRONISMO}

Os métodos de sincronismo podem ser classificados como métodos em malha fechada (Deckmann et alli, 2003; KarimiGhartemani e Iravani, 2004; Lee et alli, 1999; Sasso et alli, 2002) ou em malha aberta (Camargo e Pinheiro, 2006; Kennel et alli, 2003; Marques, 1998; Svensson, 2001). Nos métodos em malha fechada, o ângulo de sincronismo é obtido através de uma estrutura em malha fechada para sincronizar o valor estimado do ângulo de fase com seu valor real. Por outro lado, métodos de sincronismo em malha aberta são simples, uma vez que eles não usam sensores mecânicos ou métodos de estimação de posição ou velocidade. O ângulo de sincronismo ou o vetor normalizado de sincronismo é obtido diretamente das tensões alternadas (Marques, 1998; Svensson, 2001) ou das tensões estimadas (Kennel et alli, 2003). Uma vez que geradores de indução com rotor em gaiola de esquilo são economicamente recomendados para aplicações de baixa potência em locais isolados (Simões et alli, 2006; Simões e Farret, 2008), o método de sincronismo deve ser simples e não deve onerar o custo do sistema de geração. Assim, este artigo analisa quatro métodos de sincronismo em malha aberta que empregam apenas dois sensores para medir as tensões de linha nos terminais do gerador de indução, que já são medidas pelo sistema de controle para regular as tensões geradas. 


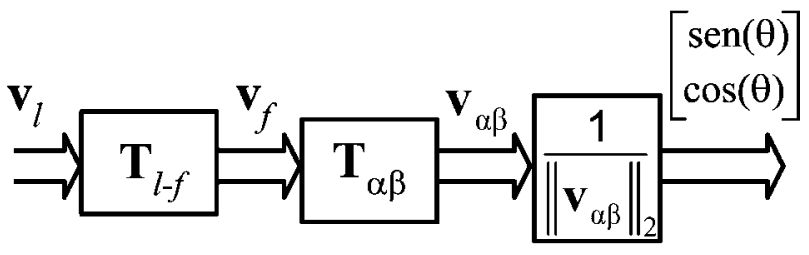

Figura 3: Diagrama de blocos do método de sincronismo I.

\subsection{Método I}

Para sistemas trifásicos a três fios, um vetor de sincronismo pode ser obtido a partir da medição de apenas duas tensões de linha (Marques, 1998). Usualmente, conversores PWM são analisados e controlados a partir de quantidades de fase (Camargo e Pinheiro, 2006), então, o vetor de tensões de linha, $\mathbf{v}_{l}$, é transformado em um vetor de tensões de fase, $\mathbf{v}_{f}$, como ilustrado na Figura 3. Considerando que a soma das tensões de fase é zero para sistemas a três fios, então:

$$
\mathbf{v}_{f}=\mathbf{T}_{l-f} \mathbf{v}_{l}
$$

onde:

$$
\begin{gathered}
\mathbf{v}_{f}=\left[\begin{array}{l}
v_{a} \\
v_{b} \\
v_{c}
\end{array}\right], \quad \mathbf{T}_{l-f}=\frac{1}{3}\left[\begin{array}{cc}
2 & 1 \\
-1 & 1 \\
-1 & -2
\end{array}\right], \\
\mathbf{v}_{l}=\left[\begin{array}{l}
v_{a b} \\
v_{b c}
\end{array}\right] .
\end{gathered}
$$

Além disso, as tensões de fase são transformadas em coordenadas $\alpha \beta$, isto é:

$$
\mathbf{v}_{\alpha \beta}=\mathbf{T}_{\alpha \beta} \mathbf{v}_{f}
$$

onde:

$$
\mathbf{v}_{\alpha \beta}=\left[\begin{array}{l}
v_{\alpha} \\
v_{\beta}
\end{array}\right] ; \mathbf{T}_{\alpha \beta}=\sqrt{\frac{2}{3}}\left[\begin{array}{ccc}
1 & -1 / 2 & -1 / 2 \\
0 & \sqrt{3} / 2 & -\sqrt{3} / 2
\end{array}\right]
$$

Um vetor normalizado de sincronismo pode ser obtido dividindo $\mathbf{v}_{\alpha \beta}$ por sua norma, isto é:

$$
\mathbf{v}_{\alpha \beta n}=\frac{\mathbf{v}_{\alpha \beta}}{\left\|\mathbf{v}_{\alpha \beta}\right\|_{2}}
$$

onde a norma Euclidiana do vetor é dada por:

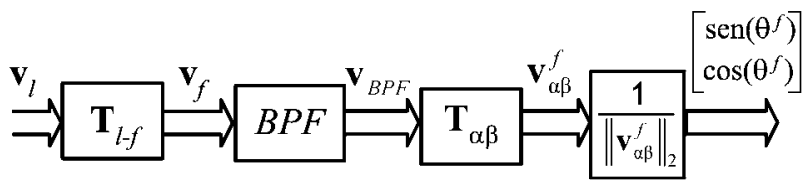

Figura 4: Diagrama de blocos do método de sincronismo II.

$$
\left\|\mathbf{v}_{\alpha \beta}\right\|_{2}=\sqrt{\left(v_{\alpha}\right)^{2}+\left(v_{\beta}\right)^{2}} .
$$

Os dois componentes do vetor $\mathbf{v}_{\alpha \beta n}$, obtidos a partir de (6), podem ser os sinais cosseno e seno usados para sincronizar o inversor PWM com as tensões produzidas nos terminais do gerador:

$$
\cos (\theta)=v_{\alpha n} \mathrm{e} \operatorname{sen}(\theta)=v_{\beta n}
$$

\subsection{Método II}

As tensões de saída de um gerador de indução auto-excitado podem apresentar harmônicos, que podem distorcer os sinais de sincronismo (Lee et alli, 1999) e também as tensões produzidas pelo sistema de geração. Para evitar esta distorção, o vetor das tensões de fase $\left(\mathbf{v}_{f}\right)$ é filtrado por filtros passabanda (BPF - Band-Pass Filters) sintonizados na freqüência fundamental, como mostrado na Figura 4. Para estes casos, os filtros passa-banda devem possuir ganho unitário e não podem defasar os sinais filtrados na freqüência fundamental.

$\mathrm{O}$ vetor das tensões de fase filtradas $\left(\mathbf{v}_{B P F}\right)$ é transformado em um vetor de tensões filtradas no sistema de coordenadas $\alpha \beta\left(\mathbf{v}_{\alpha \beta}^{f}\right)$, usando (4) e (5). Um vetor de sincronismo normalizado $\mathbf{v}_{\alpha \beta n}^{f}$ é obtido ao dividir $\mathbf{v}_{\alpha \beta}^{f}$ por sua norma Euclidiana (7). Os componentes do vetor $\mathbf{v}_{\alpha \beta n}^{f}$ são os sinais cosseno e seno para sincronizar o inversor PWM. Devido a filtragem realizada pelos filtros passa-banda, os sinais de sincronismo normalizados apresentam apenas a frequiência fundamental.

\subsection{Método III}

Cargas desequilibradas podem produzir tensões desbalanceadas nos terminais do gerador. Estas tensões desbalanceadas podem distorcer os sinais de sincronismo e, portanto, podem afetar o desempenho do sistema de controle digital.

Para evitar distorções nos sinais de sincronismo devido a tensões desbalanceadas, o vetor de sincronismo $\mathbf{v}_{\alpha \beta}+$ é alinhado com o vetor das tensões de seqüência positiva, que pode ser obtido a partir da seguinte matriz de transformação: 


$$
\mathbf{T}_{+}=\frac{1}{3}\left[\begin{array}{ccc}
1 & a & a^{2} \\
a^{2} & 1 & a \\
a & a^{2} & 1
\end{array}\right]
$$

onde $a$ é o operador de defasagem de $120^{\circ}$, representado pelo seguinte número imaginário:

$$
a=-\frac{1}{2}+j \frac{\sqrt{3}}{2} .
$$

Então, para obter o vetor das tensões de seqüência positiva no sistema de coordenadas $\alpha \beta$, deve-se pré-multiplicar tanto a parte real quanto a parte imaginária da matriz de seqüência positiva pela matriz de transformação $\mathbf{T}_{\alpha \beta}$, de tal forma que:

$$
\mathbf{v}_{\alpha \beta+}=\left[\mathrm{T}_{\alpha \beta} \operatorname{Re}(\mathbf{T}+)+j \mathrm{~T}_{\alpha \beta} \operatorname{Im}\left(\mathbf{T}_{+}\right)\right] \mathbf{v}_{f} .
$$

Para implementar o operador $j$ pode-se empregar um conjunto de filtros passa-tudo (APF - All-Pass Filters) de $1^{a}$ ordem, como mostrado na Figura 5 , projetados para possuir ganho unitário e defasagem de $90^{\circ}$ na frequiência fundamental (Lee et alli, 1999; Karimi-Ghartemani e Iravani, 2004). Então, de acordo com a Figura 5, o vetor $\mathbf{v}_{\alpha \beta+}$ é dado por:

$$
\mathbf{v}_{\alpha \beta+}=\mathbf{M}_{2} \mathbf{v}_{f}+\mathbf{M}_{1} \mathbf{v}_{f i l_{-} f}
$$

onde as matrizes $\mathbf{M}_{1}$ e $\mathbf{M}_{2}$ são:

$$
\begin{aligned}
& \mathbf{M}_{1}=\mathbf{T}_{\alpha \beta} \operatorname{Im}\left(\mathbf{T}_{+}\right)=\left[\begin{array}{ccc}
0 & \frac{\sqrt{2}}{4} & -\frac{\sqrt{2}}{4} \\
-\frac{\sqrt{6}}{6} & \frac{\sqrt{6}}{12} & \frac{\sqrt{6}}{12}
\end{array}\right] \\
& \mathbf{M}_{2}=\mathbf{T}_{\alpha \beta} \operatorname{Re}\left(\mathbf{T}_{+}\right)=\left[\begin{array}{ccc}
\frac{\sqrt{6}}{6} & -\frac{\sqrt{6}}{12} & -\frac{\sqrt{6}}{12} \\
0 & \frac{\sqrt{2}}{4} & -\frac{\sqrt{2}}{4}
\end{array}\right]
\end{aligned}
$$

Um vetor de sincronismo normalizado $\mathbf{v}_{\alpha \beta+n}$ pode ser obtido ao dividir $\mathbf{v}_{\alpha \beta+}$, obtido a partir de (12), pela sua norma Euclidiana (7). Novamente, os componentes do vetor $\mathbf{v}_{\alpha \beta+n}$ são os sinais cosseno e seno necessários para sincronizar o inversor PWM.

É importante destacar que os sinais de sincronismo são calculados a partir das tensões de seqüência positiva e, então, as tensões de seqüência negativa produzidas por cargas desequilibradas não afetam os sinais de sincronismo.

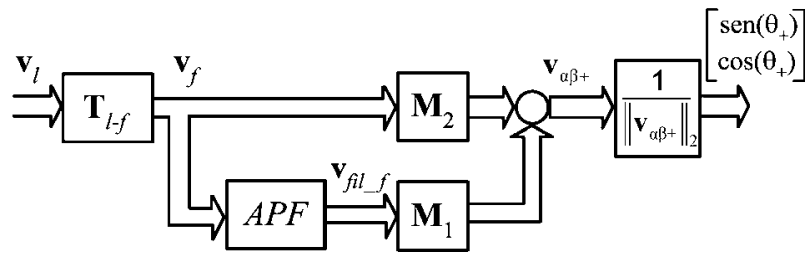

Figura 5: Diagrama de blocos do método de sincronismo III.

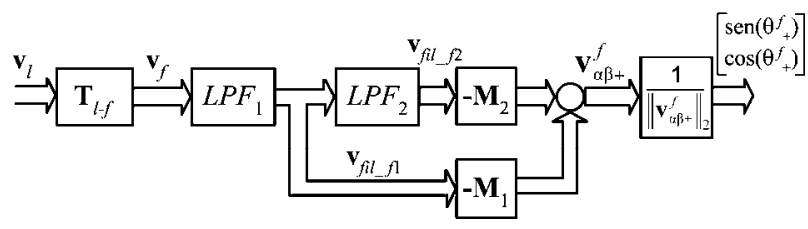

Figura 6: Diagrama de blocos do método de sincronismo IV.

\subsection{Método IV}

Outro método de sincronismo, chamado neste trabalho de método IV, pode ser usado para obter sinais de sincronismo com baixa distorção harmônica total (THD - Total Harmonic Distortion), mesmo com distorções harmônicas e cargas desequilibradas (Camargo e Pinheiro, 2006). No método de sincronismo IV, apresentado na Figura 6, o vetor das tensões de fase é filtrado por um conjunto de filtros passa-baixa (LPF - Low-Pass Filters) de $2^{a}$ ordem, $\mathrm{LPF}_{1}$, projetados para possuir ganho unitário e defasagem de $-90^{\circ}$ na frequiência fundamental.

Logo, este vetor está deslocado $-90^{\circ}$ na frequiência fundamental com relação ao vetor $\mathbf{v}_{f}$. $\mathrm{O}$ vetor filtrado $\mathbf{v}_{f i l} f 1$ é novamente filtrado por um conjunto de filtros passa-baixas chamado de $L P F_{2}$, um para cada tensão de fase. Estes promovem um deslocamento de fase adicional de $-90^{\circ}$ resultando no vetor filtrado $\mathbf{v}_{f i l \_f 2}$, como mostrado na Figura 6 , que apresenta a mesma amplitude na freqüência fundamental do vetor original $\mathbf{v}_{f}$, mas com uma defasagem de $-180^{\circ}$. Assim, as matrizes $\mathbf{M}_{1}$ e $\mathbf{M}_{2}$ devem ser multiplicadas por um ganho unitário negativo. Dessa forma, o vetor de sincronismo estará alinhado com o vetor de seqüência positiva das tensões de fase filtradas.

Então, a partir da Figura 6, o vetor de sincronismo $\mathbf{v}_{\alpha \beta+}^{f}$ pode ser expresso como:

$$
\mathbf{v}_{\alpha \beta+}^{f}=-\mathbf{M}_{2} \mathbf{v}_{f i l_{-} f 2}-\mathbf{M}_{1} \mathbf{v}_{f i l_{-} f 1} .
$$

Um vetor de sincronismo normalizado pode ser obtido ao dividir $\mathbf{v}_{\alpha \beta+}^{f}$ por sua norma Euclidiana (7). Os componentes do vetor de sincronismo normalizado são os sinais cosseno e seno usados para sincronizar o inversor PWM. 
De forma similar ao método III, os sinais de sincronismo são obtidos a partir do vetor das componentes de seqüência positiva das tensões de fase filtradas e, portanto, as tensões de seqüência negativa causadas por cargas desequilibradas e distorções harmônicas produzidas por cargas não lineares não aumentarão a THD dos sinais de sincronismo.

\section{ANÁLISE COMPARATIVA}

As principais características dos métodos de sincronismo descritos na seção anterior são sumarizadas na Tabela 1. Os métodos II, III e IV empregam diferentes estruturas de filtros para rejeitar harmônicos e/ou desequilíbrios (Lee et alli, 1999; Karimi-Ghartemani e Iravani, 2004; Camargo e Pinheiro, 2006). Contudo, esses filtros são projetados para a frequiência fundamental, e variações na frequiência das tensões geradas podem degradar o desempenho desses métodos.

Tabela 1: Comparação dos métodos de sincronismo.

\begin{tabular}{|c|c|c|c|c|}
\hline & $\begin{array}{c}\text { Método } \\
\text { I }\end{array}$ & $\begin{array}{c}\text { Método } \\
\text { II }\end{array}$ & $\begin{array}{c}\text { Método } \\
\text { III }\end{array}$ & $\begin{array}{c}\text { Método } \\
\text { IV }\end{array}$ \\
\hline $\begin{array}{c}\text { Rejeição de } \\
\text { harmônicos }\end{array}$ & & Sim & & Sim \\
\hline $\begin{array}{c}\text { Rejeição de } \\
\text { desequilí- } \\
\text { brios }\end{array}$ & & Sim & Sim \\
\hline $\begin{array}{c}\text { Sensibilidade } \\
\text { à variação de } \\
\text { frequiência }\end{array}$ & & Sim & Sim & Sim \\
\hline
\end{tabular}

Para quantificar esse e outros efeitos, o sistema de geração mostrado na Figura 1 foi simulado com a ferramenta SimPower Systems do Matlab/Simulink ${ }^{\circledR}$. A Tabela 2 apresenta os principais parâmetros da máquina de indução utilizada para obter os resultados de simulação.

A reatância de magnetização não é constante e seu valor depende da corrente de magnetização. A relação entre a reatância de magnetização $X_{M}$ e a corrente de magnetização $I_{M}$ foi obtida experimentalmente e é dada por:

$$
\begin{gathered}
X_{M}\left(I_{M}\right)=-0,0099 I_{M}^{4}+0,274 I_{M}^{3}-2,713 I_{M}^{2} \\
+9,357 I_{M}+15,821 .
\end{gathered}
$$

O sistema empregado para o controle do gerador de indução, apresentado na Figura 2, possui duas malhas de controle, que controlam a amplitude das tensões de saída do gerador e a amplitude da tensão do barramento. Uma vez que o sistema de geração foi projetado para operar com uma carga resistiva

\begin{tabular}{|c|c|}
\hline Potência nominal & $5 \mathrm{cv}$ \\
\hline Tensão nominal & $\begin{array}{c}220 \mathrm{~V} \\
\text { (conectado em } \\
\Delta)\end{array}$ \\
\hline Velocidade nominal & $1730 \mathrm{RPM}$ \\
\hline Freqüência nominal & $60 \mathrm{~Hz}$ \\
\hline Resistência do estator & $0,44 \Omega$ \\
\hline Resistência do rotor & $0,43 \Omega$ \\
\hline $\begin{array}{l}\text { Reatância de dispersão do } \\
\text { estator }\end{array}$ & $0,83 \Omega$ \\
\hline Reatância de dispersão do rotor & $0,83 \Omega$ \\
\hline $\begin{array}{c}\text { Resistência de perdas mecânicas } \\
\text { e no ferro }\end{array}$ & $232,3 \Omega$ \\
\hline Inércia do rotor & $0,03 \mathrm{~kg} \cdot \mathrm{m}^{2}$ \\
\hline
\end{tabular}

Tabela 2: Parâmetros do gerador de indução.

trifásica equilibrada de $3 \mathrm{~kW}$, é importante analisar a regulação dessas variáveis com a variação de carga.

A Figura 7 mostra os valores eficazes das tensões de linha produzidas pelo gerador de indução, comparando os quatro métodos de sincronismo apresentados neste artigo. O gerador inicialmente opera a vazio e, em 1,7 s, uma carga resistiva trifásica equilibrada de $3 \mathrm{~kW}$ é conectada ao gerador. Percebe-se que, ao conectar a carga, todas as tensões de linha são reguladas em $220 \mathrm{~V}$ após o regime transitório. Para a operação a vazio, as tensões geradas com os métodos II, III e IV não são reguladas exatamente em $220 \mathrm{~V}$, apresentando um erro estático muito pequeno, menor que $1 \%$ do valor nominal. Este pequeno erro em regime permanente existe porque a freqüência das tensões geradas varia com a mudança da potência ativa consumida pelas cargas. Acionando o gerador de indução com uma velocidade de 1856 RPM, a freqüência das tensões geradas é $60 \mathrm{~Hz}$ para uma carga que consome uma potência de $3 \mathrm{~kW}$, mas é $61,8 \mathrm{~Hz}$ para a operação a vazio. Esta variação de freqüência introduz uma defasagem indesejada aos sinais de sincronismo obtidos nos métodos II, III e IV, a qual poderia ser facilmente resolvida com a introdução de um método de adaptação dos parâmetros dos filtros conforme proposto por Camargo e Pinheiro, 2006. Porém, como a variação de freqüência não introduz erros significativos na regulação das variáveis de interesse, esta questão foi desprezada, a fim de atender o critério de simplicidade estrutural dos métodos de sincronismo utilizados.

De forma similar, a Figura 8 apresenta a tensão do barramento CC do inversor, utilizando diferentes métodos de sincronismo no sistema de controle. Novamente, a tensão do barramento é regulada em $450 \mathrm{~V}$ com todos os métodos de 


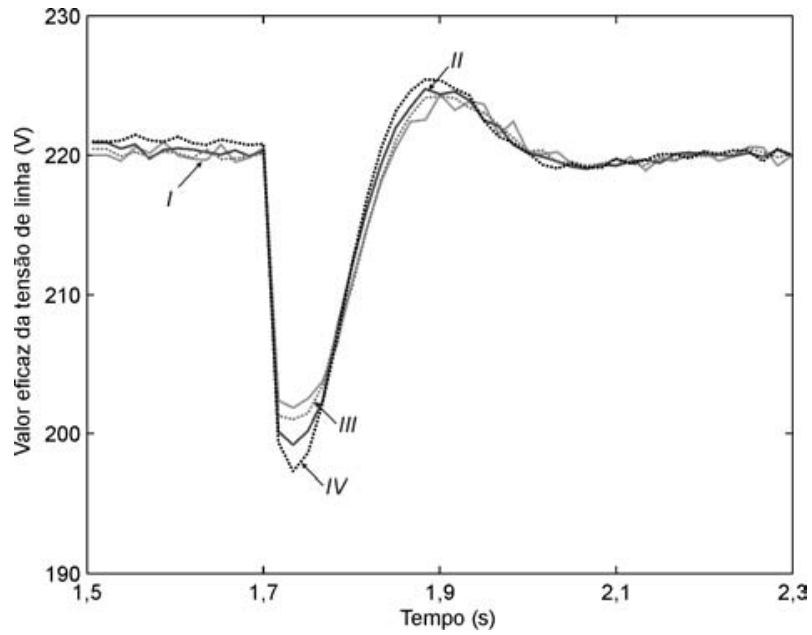

Figura 7: Valor eficaz das tensões de linha nos terminais do gerador de indução sob variação de carga: operação a vazio para carga resistiva de $3 \mathrm{~kW}$.

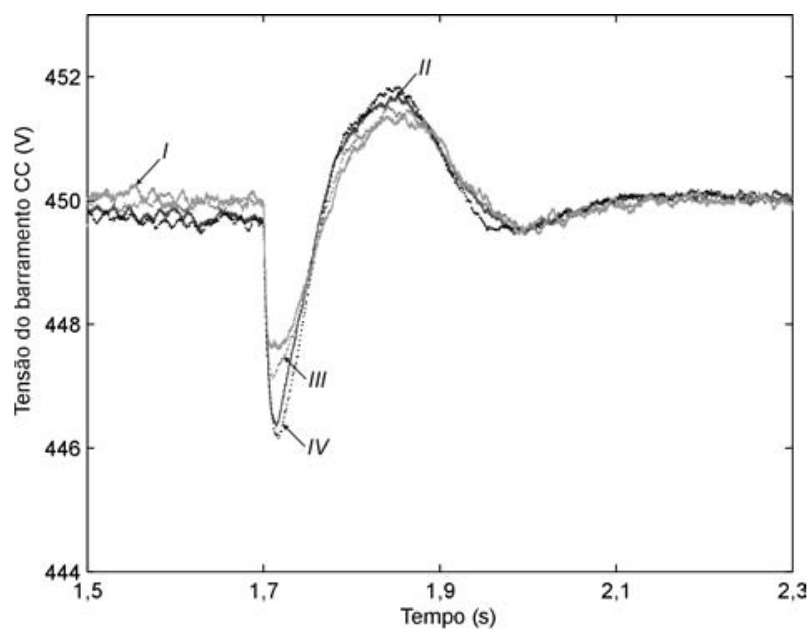

Figura 8: Tensão do barramento CC sob variação de carga: operação a vazio para carga resistiva trifásica de $3 \mathrm{~kW}$.

sincronismo para a carga de $3 \mathrm{~kW}$. Na operação a vazio existe um pequeno erro estático, menor que $0,2 \%$ do valor desejado da tensão do barramento CC, usando os métodos II, III e IV.

Além da análise da regulação estática das variáveis de interesse, deve-se comparar a qualidade das tensões produzidas pelo sistema de geração com os diferentes métodos de sincronismo. A Figura 9 mostra as formas de onda dos sinais de sincronismo (superior) e as tensões de linha nos terminais do gerador de indução (inferior) quando uma carga resistiva monofásica de $1 \mathrm{~kW}$ é conectada entre dois terminais do gerador de indução. Verifica-se que os sinais de sincronismo e, conseqüentemente, as tensões geradas (THD =3,4\%) apresentam distorções significativas, que são causadas pelo método
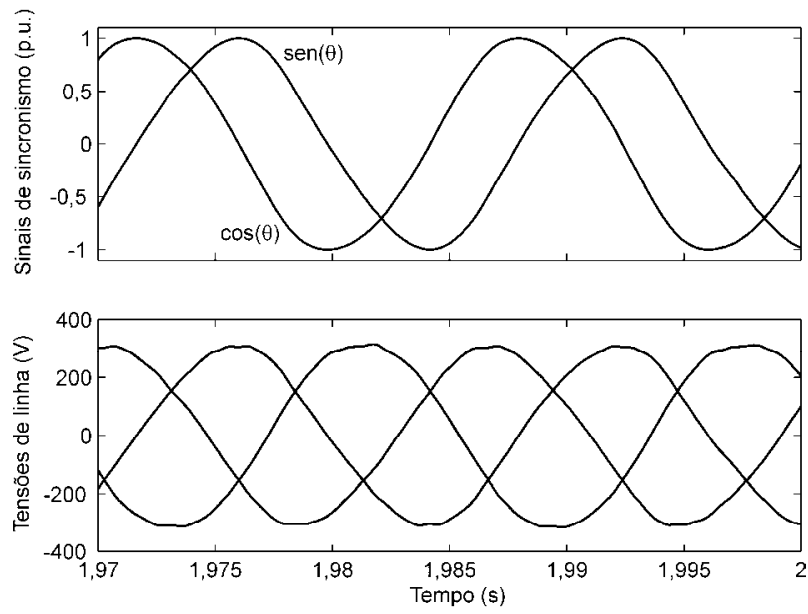

Figura 9: Desempenho em regime permanente do sistema de geração com carga desequilibrada usando o método de sincronismo I: sinais de sincronismo (superior) e tensões de linha nos terminais do gerador de indução (inferior).
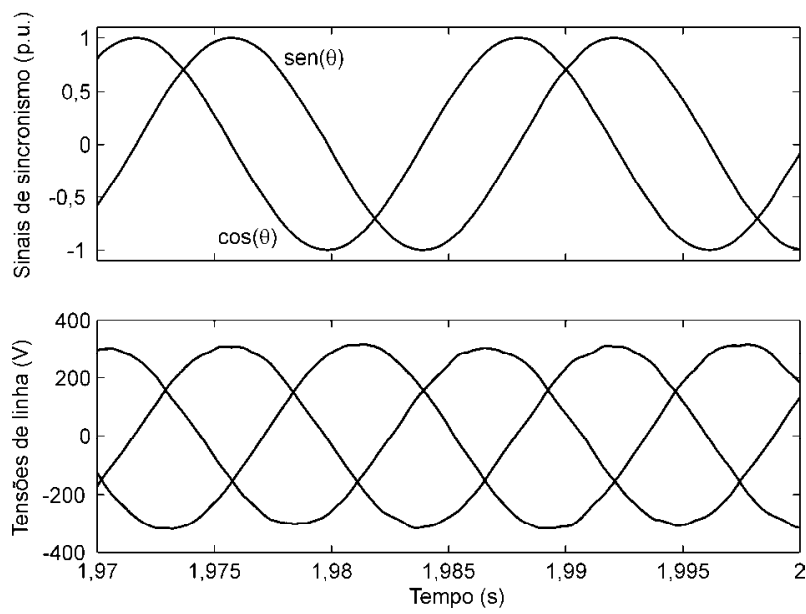

Figura 10: Desempenho em regime permanente do sistema de geração com carga desequilibrada usando o método de sincronismo III: sinais de sincronismo (superior) e tensões de linha nos terminais do gerador de indução (inferior).

de sincronismo I.

Por outro lado, o método de sincronismo III usa somente as componentes de seqüência positiva das tensões geradas e, portanto, os sinais de sincronismo não ficarão distorcidos com cargas desequilibradas. A Figura 10 apresenta um resultado para a mesma carga desequilibrada usada na simulação anterior, mas agora utilizando o método III. Nota-se que os sinais de sincronismo apresentam distorções menores que as apresentadas ao usar o método I, assim, a THD das tensões geradas foi reduzida para $1,5 \%$. 


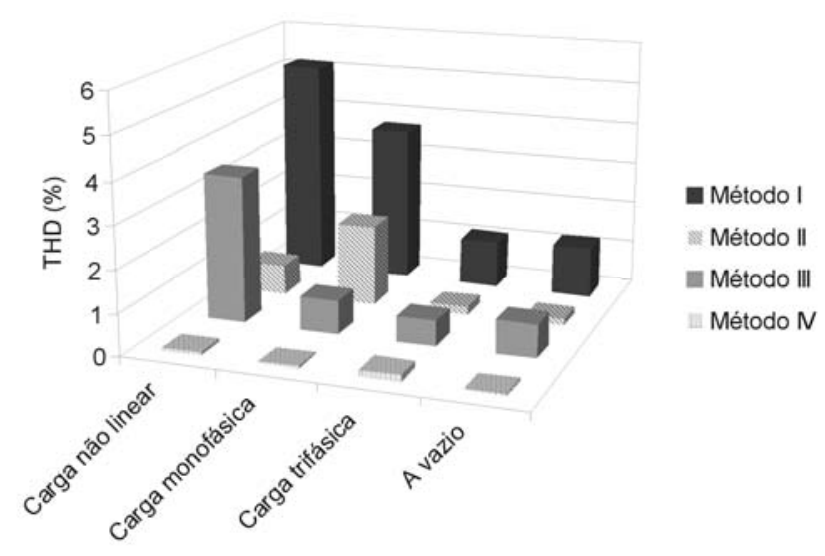

Figura 11: THD do sinal seno de sincronismo.

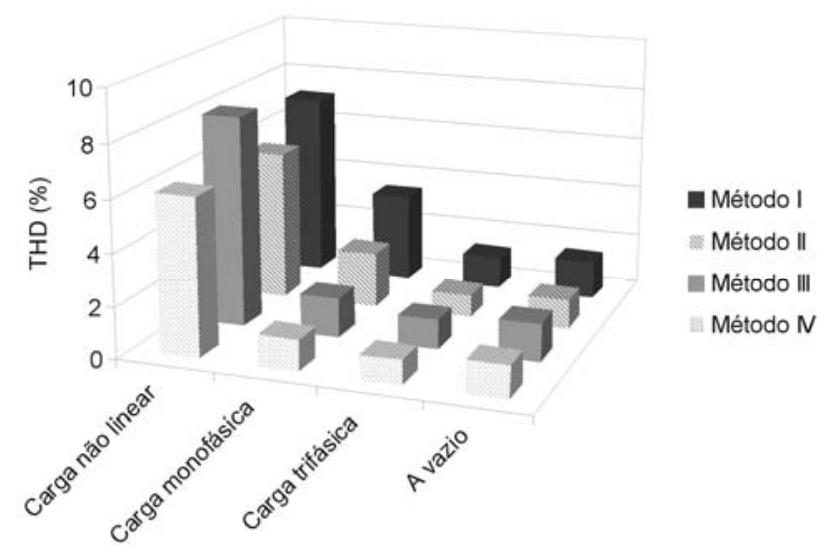

Figura 12: THD das tensões de linha nos terminais do gerador de indução.

Outras simulações foram realizadas para diversas condições de carga e seus resultados podem ser comparados nas Figuras 11, 12 e 13. A Figura 11 mostra claramente que o sinal seno de sincronismo gerado pelo método I apresenta uma THD significativa para cargas não lineares e desequilibradas. Como mostrado na Tabela 1, é possível reduzir a THD dos sinais de sincronismo para cargas não lineares ao usar o método II ou o método IV. Para cargas desequilibradas, é possível reduzir a distorção dos sinais de sincronismo ao empregar o método III ou o método IV.

A geração de sinais de sincronismo com pequenas distorções reflete em tensões geradas com menor THD, como pode ser visualizado na Figura 12. A THD das tensões de linha produzidas pelo sistema de geração com cargas monofásicas é reduzida ao utilizar o método III ou o método IV. Por outro lado, para cargas não lineares, as tensões nos terminais do gerador são menos distorcidas ao usar o método II ou o método IV, contudo esta redução não é muito significante. Uma importante diminuição da THD para cargas não linea-

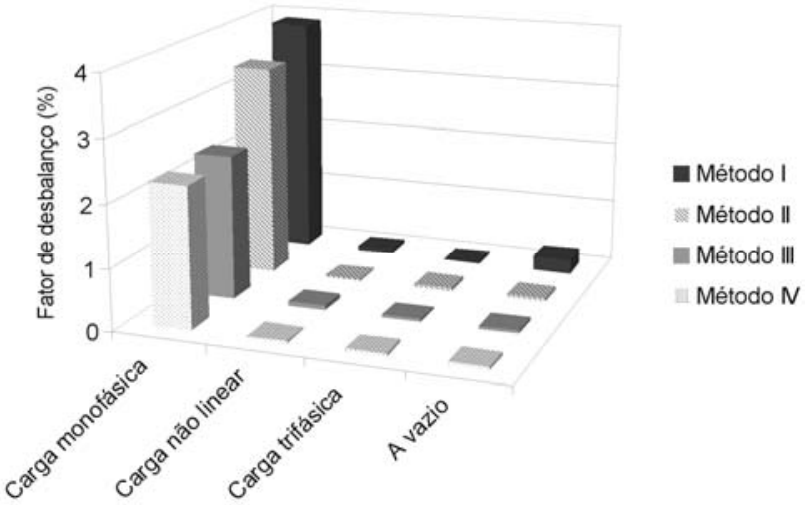

Figura 13: Fator de desbalanço das tensões de linha nos terminais do gerador de indução.

res pode ser alcançada usando o método II ou o método IV, e substituindo os controladores PI de corrente por controladores baseados na teoria do modelo interno, que minimizam os erros de rastreamento para distúrbios periódicos (Rech et alli, 2003).

A Figura 13 mostra que o fator de desbalanço (Fd) das tensões de linha (IEEE Std 141, 1993) ao aplicar uma carga monofásica nos terminais do gerador de indução é reduzido utilizando os métodos de sincronismo III ou IV ao invés do método I.

\section{RESULTADOS EXPERIMENTAIS}

Um protótipo foi construído em laboratório para realizar testes experimentais no sistema de geração apresentado na Figura 1. Os parâmetros do gerador são os mesmos usados para obter os resultados de simulação. O sistema de controle digital foi implementado no DSP TMS320F2812 da Texas Instruments. Embora tenham sido obtidos resultados experimentais com todos os métodos de sincronismo comparados neste trabalho, por questões de espaço, esta seção mostra os resultados obtidos com o método de sincronismo I, que é o método mais simples, e com o método IV, que apresentou os melhores resultados sob diferentes condições de carga.

A Figura 14 apresenta as formas de onda das tensões de linha nos terminais do gerador de indução antes de conectar o inversor PWM para regular as tensões de saída. Pode-se observar que as formas de onda das tensões produzidas pelo gerador estão distorcidas (THD $=2,1 \%$ ) e isto afetará aqueles métodos de sincronismo que são sensíveis a harmônicos. Este efeito pode ser verificado na Figura 15, que mostra as tensões de linha nos terminais do gerador ao empregar o método de sincronismo I. A Figura 15(a) mostra as tensões de linha ( $\mathrm{THD}=3,2 \%$ e $\mathrm{Fd}=0,5 \%$ ) ao conectar uma carga resistiva trifásica equilibrada de $3 \mathrm{~kW}$. A Figura 15(b) apre- 


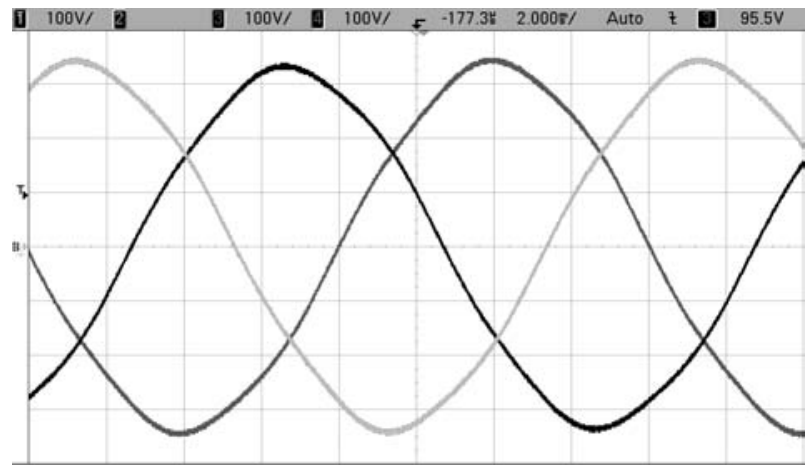

Figura 14: Resultado experimental: tensões de linha nos terminais do gerador de indução antes de conectar o inversor PWM.

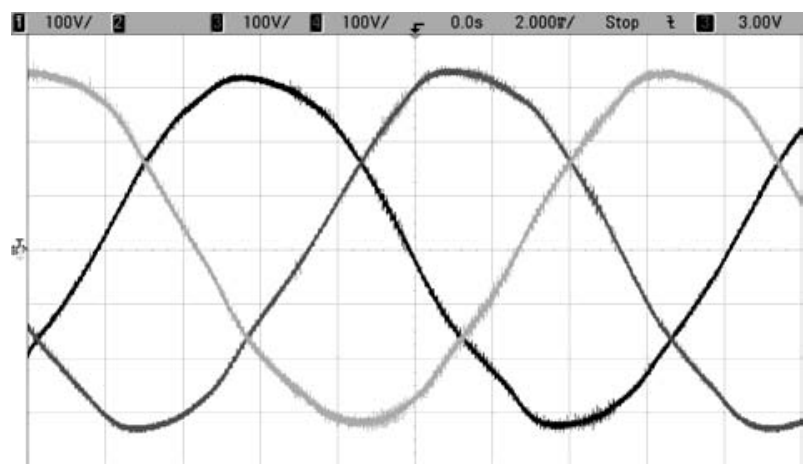

(a)

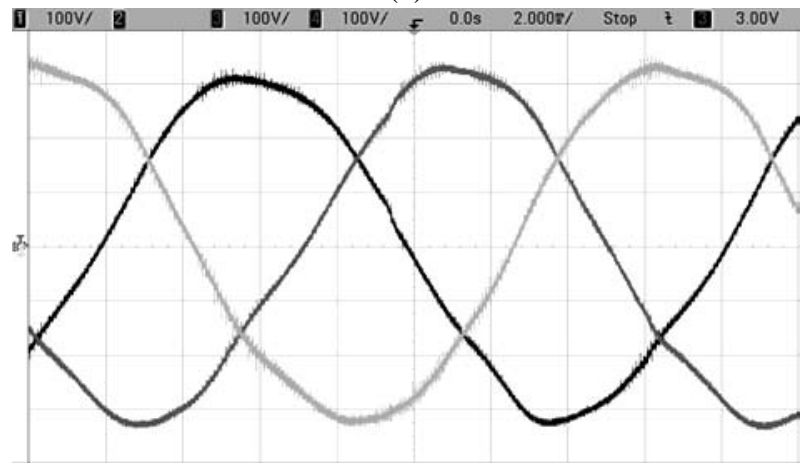

(b)

Figura 15: Resultado experimental: tensões de linha nos terminais do gerador de indução usando o método de sincronismo I. (a) Carga resistiva trifásica equilibrada de $3 \mathrm{~kW}$. (b) Carga resistiva monofásica de $1 \mathrm{~kW}$.

senta as formas de onda das tensões de linha (THD $=4,6 \%$ e $\mathrm{Fd}=2,6 \%$ ) com uma carga resistiva monofásica de $1 \mathrm{~kW}$, aumentando a distorção nas tensões geradas devido a carga desequilibrada.

Por outro lado, a Figura 16 mostra a resposta em regime permanente do sistema de geração ao usar o método de sincro-

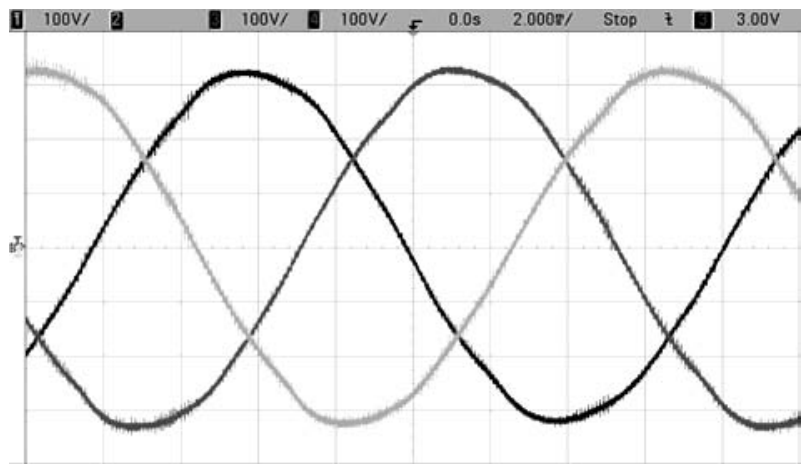

(a)

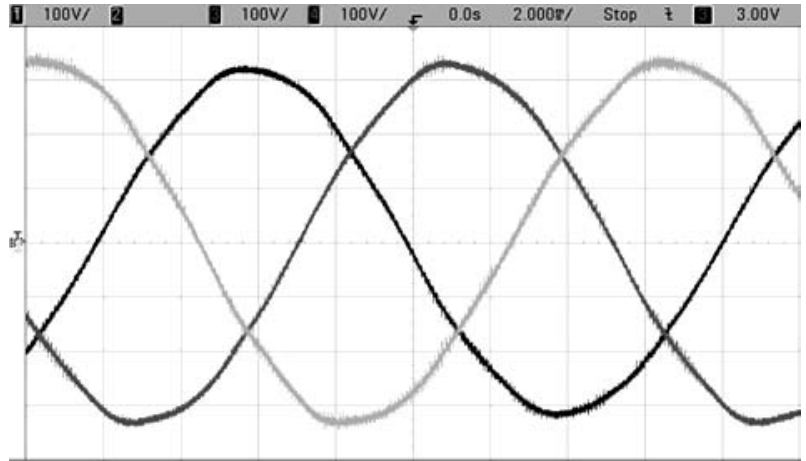

(b)

Figura 16: Resultado experimental: tensões de linha nos terminais do gerador de indução usando o método de sincronismo IV. (a) Carga resistiva trifásica equilibrada de $3 \mathrm{~kW}$. (b) Carga resistiva monofásica de $1 \mathrm{~kW}$.

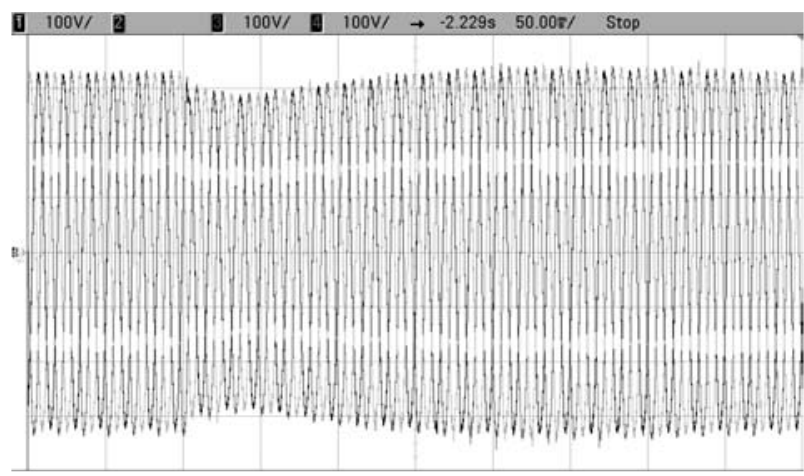

Figura 17: Resultado experimental: resposta transitória do sistema de geração usando o método de sincronismo IV.

nismo IV. A Figura 16(a) apresenta as tensões de saída (THD $=1,8 \%$ e $\mathrm{Fd}=0,3 \%$ ) com uma carga resistiva trifásica equilibrada de $3 \mathrm{~kW}$, enquanto que a Figura 16(b) mostra as formas de onda das tensões de linha (THD $=2 \%$ e $\mathrm{Fd}=2 \%$ ) com a mesma carga desequilibrada usada para obter o resultado apresentado na Figura 15(b). Em ambos os casos, é possível verificar uma significativa redução na THD ao usar o método 
IV.

Por fim, a Figura 17 apresenta as formas de onda das tensões de linha nos terminais do gerador de indução para um degrau brusco de carga de operação a vazio para uma carga resistiva trifásica de $3 \mathrm{~kW}$. Observa-se que a resposta transitória do sistema de controle é satisfatória mesmo com este degrau severo de carga.

\section{CONCLUSÕES}

Este artigo apresentou uma avaliação de diferentes métodos de sincronismo em malha aberta para serem aplicados em geradores de indução auto-excitados sob diversas condições de carga. Estes métodos de sincronismo foram escolhidos porque os mesmos apresentam uma significativa redução de custo se comparados com aqueles métodos que empregam sensores mecânicos para medir posição ou velocidade. Mesmo com a variação da frequiência, causada pela mudança da potência ativa consumida pelas cargas, os métodos de sincronismo empregados no sistema de geração resultaram em uma boa regulação das tensões de saída e da tensão do barramento CC. Contudo, os resultados incluídos no artigo mostram que o impacto destes métodos na THD e no fator de desbalanço das tensões produzidas pelo sistema de geração é significativo.

Os métodos de sincronismo apresentados neste artigo diferem em termos de complexidade e desempenho. O método I é muito simples, resultando em um reduzido tempo computacional para a sua implementação, mas os sinais de sincronismo são distorcidos para cargas não lineares ou desequilibradas. Assim, não é possível gerar tensões com baixa THD, mesmo empregando controladores com ótimo desempenho. Por outro lado, o método IV requer um maior tempo de cálculo que os tempos gastos pelos demais métodos investigados neste artigo, mas os sinais de sincronismo e, portanto, as tensões geradas apresentam uma menor THD, mesmo na presença de cargas desequilibradas e não lineares.

\section{AGRADECIMENTOS}

Os autores agradecem o Departamento Municipal de Energia de Ijuí (DEMEI) e o Conselho Nacional de Desenvolvimento Científico e Tecnológico (CNPq) pelo suporte financeiro. Agradecimentos especiais a Metalcorte Motores e a EPCOS pelas doações de equipamentos. Por fim, os autores agradecem os acadêmicos Andressa Regina Feyh e Guilherme Sebastião da Silva pela ajuda na implementação do protótipo.

\section{REFERÊNCIAS}

Ahmed, T., Nishida, K., Nakaoka, M. (2006). Advanced control of PWM converter with variable-speed induction generator. IEEE Trans. Ind. Applicat., 42(4): 934-945.

Basset, D. E., Potter, M. F. (1935). Capacitive excitation for induction generators. AIEE Transactions, 54: 540-545.

Camargo, R. F., Pinheiro, H. (2005). Comparison of six digital current control techniques for three-phase voltagefed PWM converters connected to the utility grid. IEEE PESC Conf. Rec.: 1422-1428.

Camargo, R. F., Pinheiro, H. (2006). Synchronization method for three-phase PWM converters under unbalanced and distorted grid. IEE Proc. - Electr. Power Appl., 153(5): 763-772.

Cichowlas M., Kazmierkowski, M. (2002). Comparison of current control techniques for PWM rectifiers. IEEE ISIE Proc.: 1259-1263.

Dai, K., Liu, P., Xiong, J., Chen, J. (2003). Comparative study on current control, for three-phase SVPWM voltage-source converter in synchronous rotating frame using complex vector method. IEEE PESC Conf. Rec.: 695-700.

Deckmann, S. M., Marafão, F. P., Pádua, M. S. (2003). Single and three-phase digital PLL structures based on instantaneous power theory. Anais do COBEP: 225-230.

Erickson, R. W., Maksimovic, D. (2001). Fundamentals of Power Electronics, Segunda Edição, Kluwer Academic Publishers.

IEEE Std 141-1993 (1993). IEEE Recommended Practice for Electric Power Distribution for Industrial Plants (Red Book), IEEE.

Karimi-Ghartemani, M., Iravani, M. R. (2004). A method for synchronization of power electronic converters in polluted and variable-frequency environments. IEEE Trans. Power Systems, 19(3): 1263-1270.

Kennel, R., Linke, M., Szczupak, P. (2003). Sensorless control of 4 quadrant-rectifiers for voltage source inverters (VSI). IEEE PESC Conf. Rec.: 1057-1062.

Kuo, S.-C., Wang, L. (2001). Analysis of voltage control for a self-excited induction generator using a currentcontrolled voltage source inverter (CC-VSI). IEE Proc. - Gener., Transm., Distrib., 148(5): 431-438.

Lee, S.-J., Kang, J.-K., Sul, S.-K. (1999). A new phase detecting method for power conversion systems considering distorted conditions in power system. Conf. Rec. IEEE IAS Annual Meeting: 2167-2172. 
Leidhold, R., Garcia, G., Valla, M. I. (2002). Induction generator controller based on the instantaneous reactive power theory. IEEE Trans. Energy Conv., 17(3): 368373.

Lopes, L. A. C., Almeida, R. G. (2006). Wind-driven selfexcited induction generator with voltage and frequency regulated by a reduced rating voltage source inverter. IEEE Trans. Energy Conv., 21(2): 297-304.

Marques, G. D. (1998). A comparison of active power filter control methods in unbalanced and non-sinusoidal conditions. Proc. IEEE IECON'98: 444-449.

Marra, E. G., Pomilio, J. A. (1999). Self-excited induction generator controlled by a VS-PWM bidirectional converter for rural applications. IEEE Trans. Ind. Applicat., 35(4): 877-883.

Rech, C., Pinheiro, H., Gründling, H. A., Hey, H. L., Pinheiro, J. R. (2003). Comparison of digital control techniques for low cost PWM inverters. IEEE Trans. Power Electr., 18(1): 401-410.

Sasso, E., Sotelo, G., Ferreira, A., Watanabe, E., Aredes, M., Barbosa, P. G. (2002). Investigação dos modelos de circuitos de sincronismo trifásicos baseados na teoria de potências real e imaginária instantâneas (p-PLL e q-PLL). Anais do CBA: CD-ROM.

Simões, M. G., Chakraborty, S., Wood, R. (2006). Induction generators for small wind energy systems. IEEE Power Electronics Society Newsletter, 18(3): 19-23.

Simões, M. G., Farret, F. A. (2007). Alternative Energy Systems: Design and Analysis with Induction Generators, Segunda Edição, CRC Press.

Singh, B., Murphy, S. S., Gupta, S. (2004). Analysis and design of STATCOM-based voltage regulator for self-excited induction generators. IEEE Trans. Energy Conv., 19(4): 783-790.

Svensson, J. (2001). Synchronization methods for gridconnected voltage source converters. IEE Proc. $-\mathrm{Ge}$ ner., Transm., Distrib., 148(3): 229-235. 